\title{
Can Partial Structures Accommodate INCONSISTENT SCIENCE?
}

\author{
Peter Vickers \\ University of Leeds
}

\begin{abstract}
The semantic approach to scientific representation is now long established as a favourite amongst philosophers of science. One of the foremost strains of this approach-the model-theoretic approach (MTA)-is to represent scientific theories as families of models, all of which satisfy or 'make true' a given set of constraints. However some authors (Brown 2002, Frisch 2005) have criticised the approach on the grounds that certain scientific theories are logically inconsistent, and there can be no models of an inconsistent set of constraints. Thus it would seem that the MTA fails to represent inconsistent scientific theories at all, and this raises concerns about the way it represents in general. In a series of papers (1990, 1993, 1995) and a recent book (2003) da Costa and French have developed a variant of the MTA approach which they call 'partial structures', and which they claim can accommodate inconsistent theories. I assess this claim, looking to two theories which have been called 'inconsistent': Bohr's theory of the atom and classical electrodynamics.
\end{abstract}

Keywords: Partial structures, inconsistency, theories, representation, Bohr, classical electrodynamics.

\section{Introduction}

During the 1960s, 70s and 80s the "semantic" view took over from the "syntactic" or "received" view as the preferred method of the representation of scientific theories and models for philosophers of science. No longer were theories to be represented as partially interpreted, logically closed axiomatic systems in first order logic, as Carnap, Reichenbach and other logical empiricists suggested in the 1940s and 50s. Now they were to be represented as families, or classes, of models.

Precisely what should be meant by 'model' is still an area of open debate, although two conceptions of 'model' are central to the position. On the one hand there is the branch of the semantic view known as the model-theoretic approach (MTA), which ultimately draws on the work of Tarski and others in the 1930s and 40s. A 'model' in this sense is a structure which satisfies a set of sentences by interpreting them in such a way that they come out true. On the other hand there are 'models' in science which are meant to represent a state of affairs, such as the Met Office's model of how the weather in the London area will develop over the next 24

Principia 13(2): 233-50 (2009).

Published by NEL - Epistemology and Logic Research Group, Federal University of Santa Catarina (UFSC), Brazil. 
hours. Rather than making true a set of uninterpreted sentences, or satisfying a set of constraints, such a model is strictly speaking false, since it deviates from the actual situation in various ways by making approximations and idealizations. In short (roughly speaking), models of the first kind interpret to make true, whereas models of the latter kind represent falsely.

There are complicated stories to tell about these two conceptions of 'model', and how they relate to each other. Thomson-Jones $(2006,527-8)$ provides a helpful discussion, distinguishing between three different roles for models: (i) serious interpreters are structures which 'make true' a set of (partially) uninterpreted sentences (of first order logic, say) by interpreting them; (ii) description fitters are structures, or other types of entity, which fit an interpreted description (in the language of set theory, mathematics, natural language, etc.); (iii) mathematical models are models which represent a given system, often by means of a state-space with a trajectory defined thereon. He argues, to my mind persuasively, that the semantic approach does best to leave behind models in the sense of serious interpreters. But this still leaves the role of description fitting, and this is enough for present purposes. It is impossible for any entity to fit an inconsistent description, just as it is impossible for any structure to make true an inconsistent set of sentences (of first order logic, say). Thus our question of concern is, does the MTA-whether interpreted in terms of models as serious interpreters or mere description fitters-have the resources to accommodate inconsistent theories? ${ }^{1}$

The MTA was criticised in the 1980s for being too inflexible in certain respects. Either a structure is a model of a set of constraints or it is not, and there is no room for the kind of approximate representations and partial correspondences which are part and parcel of real science. In answer da Costa, French and others developed a variant of the approach in the 1990s, called the 'partial structures' approach. It is within this approach that the satisfaction and representation of inconsistent scientific theories is supposedly accomplished. As da Costa and French put it,

$[R]$ egarding theories in terms of partial structures offers a straightforward and natural way of accommodating inconsistency. (da Costa and French 2003, 85)

However, the semantic approach remains woefully underdeveloped on this issue, as the following discussion will reveal.

In $\S 2$ the basic concepts of the partial structures approach will be presented, and it is shown how an inconsistent set of constraints can be satisfied. The discussion is in terms of structures as 'serious interpreters', but a translation into the language of set-theory would be simple enough (cf. van Fraassen 1972, 310). In $\S 3$ the discussion is extended to inconsistent scientific theories, and I respond to Frisch's (2005) objection to the partial structure representation of classical electrodynamics.

Principia 13(2): 233-50 (2009). 
In $\S 4$ two concerns are raised, the first about how the partial structures program would accommodate different types of inconsistent theory, and the second about the professions of the semantic approach to answer the question 'What is a scientific theory?' $\S 5$ is the conclusion.

\section{Partial structures and inconsistency}

In his 2006 paper Thomson-Jones presents the following example of a truth-making structure. The sentence to be 'made true' is,

$$
\forall x(P x \rightarrow Q x)
$$

and the structure making it true is $S=\langle D, P, Q\rangle$, where $D$ is the domain over which the quantifier ranges, and $P$ and $Q$ are sets which define the predicates in the sentence. In the domain $D$ we have five things, the Spice Girls: Posh, Ginger, Baby, Sporty and Scary. In P we have only one Spice Girl, Posh. It might be supposed that Thomson-Jones' $P$ stands for ' Posh is the only member of the domain that satisfies the requirement. In $Q$ we have two elements of the domain, Posh and Ginger. It might be supposed that $Q$ stands for "_ has her birthday in a month beginning with the letter ' $\mathrm{A}$ ". Then the sentence is interpreted to say 'Take any Spice Girl: if she's married to David Beckham then her birthday is in either April or August'. This is true, so the structure is a model of the sentence; it makes it true. ${ }^{2}$

The crucial difference in the case of partial structures is the role played by the relations. Note that when we give an extension for $P$ we have only two choices for each element in the domain: either put the element in $P$ or leave it out. This makes sense if $P$ really is meant to stand for ' in the domain either she is married to David Beckham or she is not. However, sometimes we might not know whether we should put a given element of the domain in the extension of a given predicate, or we might simply want to leave it open whether a given element belongs in that extension. With partial structures this is made possible, since the extension of each predicate (or relation) has three sections, $R_{1}, R_{2}$ and $R_{3} . R_{1}$ includes those elements of the domain which belong to the predicate, $R_{2}$ includes those elements of the domain which do not belong to the predicate, and $R_{3}$ includes those elements of the domain for which we are not sure, or for which we want to leave it open. ${ }^{3}$

So, for example, suppose that we want to express the fact that we don't know for sure that Posh Spice is married to David Beckham (they could have divorced on the quiet). And we're not sure that David Beckham hasn't subsequently married Baby

Principia 13(2): 233-50 (2009). 
Spice (again, on the quiet). Then we can present the structure $S=\langle D, P, Q\rangle$ where,

$$
\begin{aligned}
& P=\langle\emptyset,\{\text { Ginger, Sporty, Scary }\},\{\text { Posh, Baby }\}\rangle \\
& Q=\langle\{\text { Posh, Ginger }\},\{\text { Sporty, Scary, Baby }, \emptyset\rangle
\end{aligned}
$$

Now $S$ gives us a partial structure for our original sentence, it partially satisfies it. The reason for this is that there is a way to partially extend the structure which would make the sentence true in the original sense; in other words there is a way one can move the elements from $R_{3}$ into $R_{1}$ or $R_{2}$ such that the structure becomes 'full' (nonpartial) and the sentence is made true in the usual sense. When $S$ is made 'full' in this way the final result is called an ' $S$-normal structure'. As da Costa and French put it,

[W] say that $\mathrm{S}$ ['some sentence'] is pragmatically [or partially] true in the structure $A$ if there exists an $A$-normal $B$ in which $S$ is true, in the correspondence sense. (da Costa and French 2003, 19)

One might also note at this stage that this definition of a partial structure allows for structures which 'make a sentence partially true', but in a completely uninformative way. For example, the following partial structure (with domain $D$ as before) also makes our sentence $\forall x(P x \rightarrow Q x)$ 'partially true':

$$
\begin{aligned}
& P=\langle\emptyset, \emptyset,\{\text { Ginger, Sporty, Scary, Posh, Baby }\}\rangle, \\
& Q=\langle\emptyset, \emptyset,\{\text { Posh, Ginger, Sporty, Scary, Baby }\} .
\end{aligned}
$$

The reason is that, as required, there is a way to partially extend the structure to make our sentence true in the traditional 'full' sense. But our structure is totally uninformative: it 'stays silent' on which elements of the domain belong to $P$ (are married to David Beckham) and which elements of the domain belong to $Q$ (have birthdays in April or August).

But this is no criticism of the partial structures approach. It merely needs to be acknowledged that there are degrees to which a given partial structure is informative. The totally uninformative structure given above can, in a loose sense, be said to make the sentence 'partially true', but it is totally uninteresting to the philosopher of science. On pp.51-2 of their book, da Costa and French discuss the relationship between two structures, and how that is a matter of degree: there is a degree of correspondence, expressible in terms of respects and degrees, depending on how many relations they have in common, and then how many elements are in $R_{3}$ in those relations. This idea can be extended to express the degree to which a given structure makes a given sentence partially true. We can have partial structures ranging from 'totally uninformative' to 'full'.

Principia 13(2): 233-50 (2009). 
Now, in its original, non-partial version it seems clear enough that the MTA cannot handle inconsistent scientific theories. Thus we find passages such as the following:

Since there are no models of inconsistent sets of sentences, straightforward semantic accounts fail. (Brown 1992, 397)

[I]f we think of a theory's models as structures in which the theory's laws or axioms are true [the MTA], then the laws of the theory need to be consistent. For a theory with inconsistent laws has no models. (Frisch 2005, 7)

However, it is equally clear that on the new approach inconsistent theories can be accommodated. For starters, we can (trivially) make an inconsistent theory partially true using a totally uninformative structure. Consider the theory made up of two sentences ' $\exists x P x^{\prime}$ ' and ' $\exists x P x$ '. This is made partially true by a structure with a domain of one element ' $a$ ', and an extension of $P$ which puts ' $a$ ' in $R_{3}$ :

$$
S=\langle D, R\rangle=\langle\{a\},\langle\emptyset, \emptyset,\{a\}\rangle\rangle
$$

This makes ' $\exists x P x$ ' partially (or pragmatically) true because there is a way to partially extend $S$ (by putting ' $a$ ' in $R_{1}$ ) such that it comes out true, and it also makes ' $\sim \exists x P x$ ' partially true because there is a way to partially extend $S$ (by putting ' $a$ ' in $R_{2}$ ) such that it comes out true. Of course we can't make both true at the same time, but that is something the advocate of partial structures is happy to concede. Indeed, that is one of the motivations for partial structures. ${ }^{4}$

Several questions now follow. Can informative structures make inconsistent theories partially true? Can they make them partially true in an interesting and revealing way? And what special considerations arise when we move from 'toy' examples like the one just given to the statements of scientific theories?

\section{Application to scientific theories}

Two theories are especially relevant to the present debate: classical electrodynamics and Bohr's theory of the atom. Frisch (2005) has argued that the partial structures approach does not adequately accommodate the inconsistency in CED, whereas da Costa and French (2003) have argued that partial structures do adequately accommodate the inconsistency in Bohr's theory of the atom. The two theories will be taken in turn.

\subsection{Classical electrodynamics}

Here is an example of an inconsistent 'theory'-call it CED*-with three sentences (labelled ME*, LFE* and EC* for reasons which will soon become clear), which can be made partially true by a partial structure that is largely informative:

Principia 13(2): 233-50 (2009). 


$$
\begin{aligned}
& M E * \forall x(P x \rightarrow Q x), \\
& L F E *: \forall x(Q x \rightarrow R x), \\
& E C *: \exists x(P x \wedge \sim R x) .
\end{aligned}
$$

One structure that makes $C E D^{*}$ partially true is $S=\langle D, P, Q, R\rangle$, where,

$$
\begin{aligned}
& D=\{a, b\}, \\
& P=\langle\{a, b\}, \emptyset, \emptyset\rangle, \\
& Q=\langle\{a\}, \emptyset,\{b\}\rangle, \\
& R=\langle\emptyset, \emptyset,\{a, b\}\rangle .
\end{aligned}
$$

In fact, this makes it true in a special way: depending on how you partially extend $S$ to give an $S$-normal structure, you can make any two of the three statements true. If you put everything in $R_{1}$ you make $\mathrm{ME}^{\star}$ and $\mathrm{LFE}^{*}$ true, but EC* false; if you put everything in $R_{1}$ except the ' $b$ ' in $R$, you make ME* and EC* true, but LFE* false; finally, if you put the two ' $b$ 's in $R_{2}$ (in $Q$ and $R$ ), and the ' $a$ ' in $R_{1}$ (in $R$ ), you make $\mathrm{EC}^{\star}$ and $\mathrm{LFE}^{\star}$ true, but $\mathrm{ME}^{\star}$ false.

This seems to be the appropriate way to represent how partial structures could accommodate the "inconsistency" in classical electrodynamics (CED). ${ }^{5}$ Frisch (2005) presents four statements which, he feels, represent the content of CED. The three most substantial of these are the Maxwell equations (ME), the Lorentz force equation (LFE), and energy conservation (EC). Now as Frisch presents them, these statements are inconsistent, and thus there can be no model of them in the model-theoretic sense. But the example given above shows how such a theory might be satisfied by a partial structure. Of course the logical content of the sentences I have labelled ME*, $\mathrm{LFE}^{\star}$ and EC* does not faithfully represent the equations of CED, but there doesn't seem to be any reason in principle why CED could not be represented if CED* can.

Now, Frisch is keen to reject partial structures as a suitable representative device for CED. He writes,

[T] he partial structures approach would appear to recommend that different regions of space-time which contain systems of particles and fields (i.e., different subsets of A [the domain]) satisfy some of the fundamental equations of the theory, but not all of them: Some particle-field systems satisfy the Lorentz equation of motion, while others satisfy the Maxwell equations and energy conservation, say. But this misconstrues the commitment scientists appear to have to the theory. It is not the case that we take some electrons to be governed by the Lorentz force equation and others by the Maxwell equations-our commitment to the approximate truth ... of the Maxwell-Lorentz equations, extends to all classical systems of charges and fields. (Frisch 2005, 39f.)

Principia 13(2): 233-50 (2009). 
So, the claim is, we think that the Maxwell-Lorentz equations are approximately true of all systems of charges and fields, not that they really are true of some systems of charges and fields and false of others. It isn't immediately clear that Frisch has applied partial structures here, though. If Frisch's understanding of the approach is correct then we have three options: systems of particles and fields are either true of the equations, false of them, or it is indeterminate (or left silent) whether they are true of them. Still, if Frisch has applied the approach correctly his criticism appears to hold: the attitude taken by scientists is the same towards all different systems of particles and fields.

Now, if things were as in CED*, then Frisch's criticism would not stand up. In that case it's not the case that some elements satisfy $\mathrm{ME}^{\star}$ and others satisfy LFE* (say). Instead, whether $\mathrm{ME}^{\star}$ and $\mathrm{LFE}^{\star}$ are satisfied depends on which elements are in which part of the extension of the different predicates $P, Q$ and $R$. In the structure given, our commitment to the approximate (partial) truth of the sentences can extend to every element in the domain (' $a$ ' and ' $b$ '). This seems to be exactly as Frisch would wish it, when he says that our commitment to the Maxwell-Lorentz equations should extend to all the elements in the domain (all systems of charges and fields). In fact this seems to link up nicely with what Frisch says next. He writes,

[F]or a given system we use only a proper subset of the theory's equations to model its behavior, where the choice of equations depends on what aspect of the interaction between charges and fields we are interested in. $(2005,40$, original emphasis)

Within the partial structures approach this 'choice' would be represented by the fact that, in the example of CED*, we have a choice about how we partially extend our structure, depending on which two "equations" (out of $\mathrm{ME}^{\star}, \mathrm{LFE}^{\star}$ and $\mathrm{EC}^{\star}$ ) we want to satisfy.

So are things as in CED*, with elements in the domain going in the extension of predicates within the equations, or are things as Frisch suggests, with elements of the domain going in the extension of relations which are the equations? It is crucial here to distinguish between two different ways in which the partial structures approach can be applied. Frisch adopts a state-space approach, as do many others including Pincock (2005), Suppe (1989), and Van Fraassen (1972). In the domain one puts ordered tuples representing possible states of the system which could be plotted as points in a state-space. One then defines the equations by putting elements (tuples) from this domain in the extension of the structure's relations. Typically, the elements in a given extension are decided by looking to one or another equation of the theory in question (cf. Suppe 1989, 155f.). Thus Frisch envisions a domain of particlefield states, and then a dilemma as to which states to put in the extension of which relation, where the relations represent particular equations, such as the LFE.

Principia 13(2): 233-50 (2009). 
Now, the partial structures program was developed by da Costa, French and others in the style of the CED* example. As noted above, one shouldn't use first order logic, but should instead formulate the statements of a given theory in the language of set-theory, and then construct a set-theoretical structure which satisfies the given statements (see van Fraassen 1972, 310). One can define the class of structures via a set-theoretical predicate which articulates the constraints in the set-theoretical axioms. In this approach the structures have a domain of individuals (rather than ordered tuples representing states), and relations $R_{i}$ which represent properties of, and relations between, the individuals (rather than simply representing the laws of the given theory). So, in Suppes' famous 1957 model-theoretic representation of classical mechanics the structures take the form $\langle P, T, s, m, f, g\rangle$, where $P$ is a set of particles, $T$ is an interval of real numbers representing times, $s(p, t)$ is a function which gives the position of particle $p$ at time $t, m(p)$ is a function giving the mass of particle $p, f(p, q, t)$ gives us the force of $p$ on $q$ at time $t$, and $g(p, t)$ gives other (external) forces on $p$ at time $t$. Crucially, the sets $s, m, f$ and $g$ do not represent the equations of the theory, and some of these elements feature in more than one of the 'axioms' of the theory. For example, $f(p, q, t)$ features in axioms P5, P6 and P7 (see Suppes 1957, 294). This looks much more like the CED* example than Frisch's reconstruction: compare how predicate $\mathrm{Q}$ in $\mathrm{CED}^{*}$ features in both $\mathrm{ME}^{*}$ and $\mathrm{LFE}^{*}$.

A more recent, and more relevant, example is Muller's suggested model-theoretic reconstruction of relativistic CED (Muller 2007, 254f.). Here the structure given is $S=\left\langle M, \eta, \mu, \varepsilon, m, Q, F, J, f_{\text {tot }}, f_{L}\right\rangle$, where $M$ and $\eta$ are a manifold representing spacetime, $\mu$ and $\varepsilon$ represent the medium, $m$ represents the mass of the charge-matter distribution, $Q$ represents the total charge under consideration, $F$ is a function on $M$ which represents the electromagnetic field, $J$ is a function on $M$ which represents the charge-current density, $f_{\text {tot }}$ represents the total force acting on the charge-matter density, and $f_{L}$ represents the electromagnetic force. $F$, for example, plays a role in three of the equations of CED Muller puts forward, so again one finds that the elements of the structure $S$ do not represent equations themselves, but instead play a role within equations, in the manner of the CED* example. ${ }^{6}$

So why has Frisch criticised the partial structures approach within the state-space presentation if it was developed with the set-theoretical-predicate presentation? The reason is that the two approaches are inter-translatable. In Suppes' representation of classical mechanics the functions representing the masses of the particles in the domain and the positions of the particles at different times, could be done away with by using a domain of ordered tuples representing states of the system. Instead of structures of the form $S=\langle P, T, m, s, f, g\rangle$, the information encoded in $P, T, m$ and $s$ is moved into a single domain of ordered tuples representing states of the system at times, whilst $f$ and $g$ remain as the relations to be satisfied. ${ }^{7}$ However, the lesson of inter-translatability goes both ways. The Suppesian has to appreciate that

Principia 13(2): 233-50 (2009). 
her structures could be made to look like the structures of the state-space approach criticised by Frisch, but Frisch has to appreciate that the state-space structures could equally be translated into the Suppesian style. The beauty of the latter is that it makes especially explicit options for representation which are somewhat hidden in the state-space approach. For example, one could put elements of the domain in the $R_{3}$ of $s$ or $m$ in Suppes' representation of classical mechanics, to represent our 'not knowing' or 'staying silent' on the mass or position of a given particle at a given time. Such possibilities for representation are easy to overlook when one divides a representation into 'states' and 'laws' as Frisch does. ${ }^{8}$

However, now Frisch might proceed with his objection in the following way. Looking at the CED* example, although our commitment can extend (contra Frisch) to all of the elements in the domain ( $a$ and $b$ ), we still have to have a rationale for putting $b$ in the $R_{3}$ part of the extension of $Q$, and $a$ and $b$ in the $R_{3}$ part of the extension of $R$. So we are singling out elements of the domain, and taking a different attitude to some than others. In the case of the representation of CED this would mean that, in order to present a partial structure satisfying the equations of the theory in the manner of $\mathrm{CED}^{*}$, we would have to take a different stance on the properties or relations of some particles, fields, or particle-field systems to others.

However, there doesn't seem to be anything wrong with this. Scientists working on CED in the early $20^{\text {th }}$ century didn't have exactly the same attitude to all particles, fields and particle-field systems vis-à-vis the equations of the theory. Some parts of the theory, in certain contexts, were considered suspect and problematic from the very beginning. As Frisch himself notes on p.35,

[T] he inconsistency is most plausibly seen as arising from the fact that the Lorentz force equation of motion ignores any effect that the self field of a charge has on its motion. The standard scheme treats charged particles as sources of fields and as being affected by fields-yet not by the total field, which includes a contribution from the charge itself, but only by the field external to the charge.

And scientists using the LFE knew full well that there was this problem with its formulation: this was manifested in the fact that, over the years, different versions of the LFE were devised. These include the Abraham-Lorentz equation, the 'delayed differential-difference equation', the Lorentz-Dirac equation and the 'regularized equation of motion' (see Frisch 2005, ch.3). The worries are magnified in particular situations, for example when charged particles are accelerated in synchrotrons so that they emit a great deal of radiation.

So scientists really do have a reason to treat some particles differently to others in certain situations relevant to electrodynamics. For example, the greater the acceleration of a particle the less the LFE (as Frisch presents it) can be trusted. So we

Principia 13(2): 233-50 (2009). 
might want to put one particle in the $R_{1}$ part of a relation representing the LFE, and another particle in the $R_{3}$ part, just as I have put ' $a$ ' in the $R_{1}$ part of $Q$ in CED*, and ' $b$ ' in the $R_{3}$ part. Of course the difficult job of representing CED rigorously via partial structures remains to be done, but these preliminary considerations seem to show that Frisch's objections can be answered, at least.

It should finally be emphasised that there isn't just one partial structure for CED, but a whole class of partial structures. There will be many different ways to represent the inconsistent assumptions with a partial structure, just as the particular partial structure given above is just one way to represent CED*. So, when it comes to representing the theory of CED as a whole, it isn't necessary for us to 'make a decision' as to which elements go in the $R_{3}$ part of which properties and relations. Instead there is a whole class of partial structures, with all different ways of representing the inconsistent assumptions, with all different elements in the $R_{3}$ parts of different relations. Which of these are interesting-faithful to how the relevant science was actually practiced, for example-is a question for another day.

\subsection{Bohr's theory of the atom}

Bohr's theory has long been described as an inconsistent theory. From the very beginning there was much complaint about the way the theory flew in the face of long-held physical principles. Thus von Laue remarked in 1914,

This is nonsense! Maxwell's equations are valid under all circumstances, an electron in an orbit must radiate. (cited in Jammer 1966, 86)

Von Laue refers to the way in which, according to Bohr's theory, an atom is made up of negatively charged electrons orbiting a positively charged nucleus. Since the electrons orbit the nucleus they are accelerated and should, according to CED, emit radiation. This means that they should lose energy and spiral into the nucleus. But Bohr posited that the electrons do not emit radiation whilst in their orbits, and that there is a state-the 'ground state'-closest to the nucleus from which an electron cannot possibly emit energy as radiation. As da Costa and French put it,

[W] have conflict between quantum and classical physics [in] ...the assertion that the ground state was stable, so that an electron in such a state would not radiate energy and spiral into the nucleus as determined by classical physics. This is the central inconsistency. (da Costa and French 2003, 91, original emphasis)

How is this 'inconsistency' to be accommodated? ${ }^{9}$ The clearest statement is given in French 2003. He writes,

Principia 13(2): 233-50 (2009). 


\begin{abstract}
At the time the model was proposed ... the notion of a stationary state was not understood at all, or at best, only partially, and if one were to represent Bohr's model in terms of partial structures, the stationary states would have to be located among the $R_{3}$, as relationships which had not yet been established to hold or not. Characterizing the model in this way one can accommodate the partial and conceptually "blurred" nature of the stationary states that allows for the internal "looseness of fit" between the component elements of the model. And this in turn, gives us an idea of how the model can still be said to represent: what it represents is a system that has elements of classical and quantum physics but has at its heart this poorly understood and conceptually indistinct notion. (French 2003, 1481)
\end{abstract}

As with CED, in Bohr's theory we have an element about which we are much less confident than the other parts of our theory. Thus we have a rationale for putting certain elements in the $R_{3}$ of certain relations. The theory is represented by a class of partial structures, and the MTA thereby manages to accommodate the inconsistency.

\title{
4. Two concerns
}

I wish to raise two concerns about the partial structures approach to inconsistent theories. The first is a worry about the partial structures program itself, and how it acts to represent the content of a given theory. The second is a concern about how a theory comes to be 'given' in the first place, how we should decide upon the theoretical content the partial structures program sets out to represent.

\subsection{Different types of inconsistent theory}

CED and Bohr's theory (to the extent that they are inconsistent) are particular kinds of inconsistent theory. In each case the inconsistencies were well known, from the theory's very inception, and there was one particular element of the theory which stood out as the trouble-maker: in CED it was the LFE, and in Bohr's theory is was the stationary states. We might consider what the partial structures program would have to say about two other possible types of inconsistent theory which might be found in science: (i) inconsistent theories where we don't know which part of the theory is to blame for the inconsistency, and (ii) inconsistent theories where we don't even realise they are inconsistent.

An example of the first option, where we know about the inconsistency but we can't decide upon a weak spot in our theory, would be Newtonian cosmology around the turn of the $20^{\text {th }}$ century (see Norton 1999, 2002; Vickers 2009). The theory is naturally conceived as a set of assumptions about gravity, time and space, the structure of the universe, etc. As Norton writes,

Principia 13(2): 233-50 (2009). 
At one time or another, virtually every supposition of Newtonian cosmology has been a candidate for modification in the efforts to eliminate the inconsistency. These candidates include Newton's law of gravitation, the uniformity of the matter distribution, the geometry of space and the kinematics of Newton's space and time itself. (Norton 2002, 191)

In this situation it might be objected that we have no proper rationale for putting one thing in the $R_{3}$ rather than another, and furthermore no rationale for choosing which relation to put something in the $R_{3}$ of. Which is the 'partially understood' or 'poorly understood and conceptually indistinct notion' of Newtonian cosmology analogous to the stationary states of Bohr's theory?

This objection rests on a misunderstanding of how partial structures are to be applied. When it comes to representing a theory one does not have to decide what to put "in the $R_{3}$ ", nor which relation's $R_{3}$ to make use of. The inconsistent set of assumptions define a class of partial structures, within which there will be individual structures which make use of the relations and their $R_{3}$ parts in all different ways. In the case of Newtonian cosmology, some of these structures will represent Seeliger's doubts about Newton's inverse-square law of gravitation, whereas others will represent Charlier's doubts about the homogeneity of the universe's mass distribution (see Norton 1999, 293 and 306). The decision about what to doubt is not made within the partial structures representation of the theory, but in the choice of which structure in the class of structures we decide to work with.

What about the case where we don't know that our theory is inconsistent? For example, consider Newtonian cosmology as it stood from c.1700-1895, before Seeliger wrote the paper which showed the scientific community that the theory was inconsistent (see Vickers 2009). ${ }^{10}$ During this period there was, from time to time, a strong, serious commitment to all of the assumptions in question, without any realisation that the assumptions were jointly inconsistent. How can the MTA accommodate a case such as this, where there is no obvious reason to 'go partial' with one's structures at all? If we don't know about the inconsistency, won't the advocate of the MTA attempt to apply full structures?

This can be answered by saying that partial structures are not a representational tool to be used only when we have inconsistency, or only when a theory includes 'poorly understood and conceptually indistinct' elements. Any given theory is to be represented by a class of models: for a consistent theory this class will include both full structures and partial structures, whereas for an inconsistent theory it will only include partial structures. Thus the advocate of partial structures can represent early Newtonian cosmology by a class of partial structures, without worrying that this doesn't do justice to the epistemic commitments of historical actors at the time. Partial structures can sometimes be used to represent the doubts, worries and misunderstandings of scientists, but other times they can be employed merely to

Principia 13(2): 233-50 (2009). 
represent possible doubts we could have. So, if we take a current theory such as quantum mechanics, and ask how that.would be represented in the MTA, we can say that it is represented by the class of structures, both full and partial, which satisfy the relevant constraints. Now we might be wrong to think that there are any full structures, because we might be wrong to think it is a consistent theory-we can't be one hundred percent sure that the theory is consistent. But this needn't be a criticism of the partial structures approach: on the contrary, the partial structures approach shows us how the semantic approach can provide a representation of an inconsistent theory, even when it isn't known to be inconsistent.

These considerations provide us with a response to one of the criticisms recently raised against the partial structures program. Morrison (2007) worries that we might not know what to put in the $R_{3}$ in a given case, because 'it is often not clear which relations/features are partially true' (207). In addition she asks, 'how much empirical support is required for something to be termed quasi-true?' (ibid.). The assumption is that a decision will have to be made about which elements to put in the $R_{3}$ of which relation in a given case. But if instead we see a theory as defining a class of partial structures, which will include individual structures covering all different partial representations, then these concerns dissolve. Instead the question will be why we decide to work with one individual structure rather than another, or which individual structure best represents the commitments and practices of scientists. But this doesn't constitute a criticism of the partial structures approach: the approach provides the appropriate structures within its class, and the objection reduces to the complaint that it might not be easy to decide which individual structures within the given class are the most interesting.

\subsection{When is a theory inconsistent?}

So it looks like the partial structures approach does indeed have the resources to represent inconsistent scientific theories. And it isn't hard to see how the approach could be extended to cover cases of inter-theory inconsistency, such as that between general relativity and quantum theory: an inconsistent theory of quantum gravity could be represented by a class of partial structures. However, it isn't altogether clear how much value there is in such partial structures. Advocates of the MTA often claim that the semantic approach provides 'the most appropriate representation of theories' (da Costa and French 2003, 25). It is meant, in some sense, to stand as an answer to the question 'What is a scientific theory?' (for example, this question is the opening sentence of da Costa and French (1990)). The answer is, 'A theory is (or is best represented as) a class of partial structures'. But this is to overlook crucial, more fundamental issues in our philosophical analysis of 'theory'.

First of all it should be noted that, in all variants of the MTA, the class of models

Principia 13(2): 233-50 (2009). 
is the class of all models which 'make true', or fit the description given by, a certain set of 'constraints', 'assumptions' or 'axioms'. For example, in the cases of CED and Bohr's theory given above, we have an inconsistent set of assumptions and the challenge is to represent those assumptions model-theoretically. But why are we interested in those assumptions? How do we decide which assumptions we want our model-theoretic structures to satisfy in the first place? It should be clear that this is a more fundamental question than the question of how to represent the assumptions semantically. If we make a mistake in putting together the assumptions in the first place, then all attempts to represent those assumptions with structures will build upon our original error.

It is precisely at this more fundamental level that mistakes are made with respect to CED and Bohr's theory, I want to argue. Following Frisch's 2005 claim that CED is inconsistent there was an immediate backlash, with Belot (2007) and Muller (2007) leading the way. The claim was made by these authors that the assumptions put together by Frisch were not assumptions appropriate to CED, and that they should be replaced with a consistent set of assumptions. But how should we adjudicate between Frisch, Muller and Belot? In Vickers (2008) I argue that a theory is not the kind of thing which has an absolutely decidable content. The theory can be put together in the way Frisch does, so long as one has good reasons to do so. Frisch does indeed have some reasons: he focuses on the assumptions which scientists tend to use in practice, rather than what they might believe to be the case. But, in the end, there will be many different ways to put together assumptions relevant to electromagnetic phenomena depending on what one's interests and goals are, and no single one of them ought to be called the theory above any other. For example, in the case of CED one will sometimes want to employ the LFE in one form, and sometimes in a different, contradictory form. Of course, this could be understood in terms of different classes of partial structures, but it seems more appropriate to do the work at the level of assumptions and sets of assumptions. This is more like how real science is practiced, and a convincing case has not yet been made that there is something to be gained from representing that practice in terms of classes of partial structures.

Similarly the inconsistency of Bohr's theory can be doubted, such that the value of attempts to represent the 'inconsistency of Bohr's theory' within the partial structures approach in French (2003) and da Costa and French (2003) are brought into question. Bartelborth (1989a), Hendry (1993) ànd Hettema (1995) all argue that the theory is not internally inconsistent at all. And Rutherford wrote in 1923,

For the first time, we have been given a consistent theory to explain the arrangement and motion of the electrons in the outer atom. (In Kramers and Holst 1923, p.xi)

Principia 13(2): 233-50 (2009). 
The main thought here is that Bohr's theory only makes use of electrostatics, and not electrodynamics. This allows for an electron which is held in its orbit by its attraction to the nucleus without demanding that the electron, as an accelerating charged particle, emits radiation. Compare Millikan's reconstruction of Bohr's theory in 1917:

Bohr's first assumption ... when mathematically stated takes the form:

$$
\frac{e E}{a^{2}}=(2 \pi n)^{2} m a,
$$

in which $e$ is the charge of the electron, $E$ that of the nucleus, $a$ the radius of the orbit, $n$ the orbital frequency, and $m$ the mass of the electron. This is merely the assumption that the electron rotates in a circular orbit ... The radical element in it is that it permits the negative electron to maintain this orbit or to persist in this so-called 'stationary state' without radiating energy even though this appears to conflict with ordinary electromagnetic theory. (Millikan 1917, 211f., former emphasis added)

Certainly Millikan admits an inconsistency between Bohr's theory and electromagnetic theory, but this hardly means that Bohr's theory itself is inconsistent. Indeed, Bartelborth (1989b) provides a model-theoretic representation of Bohr's theory in terms of full structures. It isn't at all clear what is to be gained by presenting Bohr's theory in such a way that it inconsistent, and then representing it in terms of a class of partial structures. ${ }^{11}$

So there is a much more fundamental question to be asked of theories before any model-theoretic techniques are employed. The question is, 'What should the content of a given theory be taken to be on a given occasion?' It seems clear that, in addition to theories being vague objects in the way that 'heaps' of sand are, there will be fundamentally different ways to put together theoretical assumptions depending on the particular investigation one is undertaking. ${ }^{12}$ For example, sometimes it will be more appropriate to focus on the assumptions which were used by scientists, rather than the ones that were believed to be true. And how one identifies the target domain of a given theory will also affect the content it is appropriate to consider. Much work remains to be done here, and this area of debate seems to be a much more appropriate context in which to ask the question 'What is a scientific theory?' than in the context of model-theoretic representation. ${ }^{13}$

\section{Conclusion}

The partial structures approach provides an intriguing method for representing scientific theories and models, but it is still in the relatively early stages of development. It does indeed seem to be able to accommodate inconsistencies in science,

Principia 13(2): 233-50 (2009). 
although it isn't yet clear that the manner in which it accommodates such science is a particularly revealing or interesting one. The position will stand or fall, in the end, depending on what it does for us when applied in detail to concrete cases. But concrete, detailed implementation of partial structures is still hard to find in the literature: Pincock (2005) is one example of attempted application, but he concludes with more questions than answers. More work in needed, and thus the jury is still out on the question of whether the approach will stand, as da Costa and French (1990, p.263) put it, as 'a useful and powerful tool in the analysis of scientific theories'. ${ }^{14}$

\section{References}

Bartelborth, T. 1989a. Is Bohr's Model of the Atom Inconsistent? In P. Weingartner and G. Schurz (eds.), Philosophy of the Natural Sciences, Proceedings of the $13^{\text {th }}$ International Wittgenstein Symposium, HPT, 220-23.

- 1989b. Kann es Rational Sein, eine Inkonsistente Theorie zu Akzeptieren? Philosophia Naturalis 26: 91-120.

- 2000. An Axiomatization of Classical Electrodynamics. Poznan Studies in the Philosophy of the Sciences and the Humanities 75: 333-51.

Belot, G. 2007. Is Classical Electrodynamics an Inconsistent Theory? Canadian Journal of Philosophy 37: 263-82.

Brown, B. 1992. Old Quantum Theory: A Paraconsistent Approach. PSA 1992, vol.2, 397411.

Brown, B. 2002. Approximate Truth: A Paraconsistent Account. In Meheus 2002, 81-103.

Da Costa, N. C. A. \& French, S. 1990. The Model-Theoretic Approach in the Philosophy of Science. Philosophy of Science 57: 248-65.

-1993. A Model-Theoretic Approach to 'Natural Reasoning'. International Studies in the Philosophy of Science 7: 177-90.

1995. Partial Structures and the Logic of the Azande. American Philosophical Quarterly 32: 325-39.

- 2003. Science and Partial Truth. Oxford: Oxford University Press.

French, S. 2003. A Model-Theoretic Account of Representation. Philosophy of Science 70: 1472-83.

Frisch, M. 2005. Inconsistency, Asymmetry, and Non-Locality. Oxford: Oxford University Press.

Hendry, R. F. 1993. Realism, History and the Quantum Theory: Philosophical and Historical Arguments for Realism as a Methodological Thesis. PhD thesis: LSE.

Hettema, H. 1995. Bohr's Theory of the Atom 1913-1923: A Case Study in the Progress of Scientific Research Programmes. Studies in History and Philosophy of Modern Physics 26: 307-23.

Jammer, M. 1966. The Conceptual Development of Quantum Mechanics. McGraw-Hill.

Meheus, J. (ed.) 2002. Inconsistency in Science. Dordrecht: Kluwer.

Mehra, J. \& Rechenberg, H. 1982. Historical Development of Quantum Mechanics. Springer.

Mickenberg, I.; da Costa, N. C. A.; Chuaqui, R. 1986. Pragmatic Truth and Approximation to Truth. Journal of Symbolic Logic 51: 201-21.

Millikan, R. A. 1917. The electron, its isolation and measurement and the determination of some of its properties. Chicago: University of Chicago Press.

Principia 13(2): 233-50 (2009). 
Morrison, M. 2007. Where Have All the Theories Gone? Philosophy of Science 74: 195-228.

Muller, F. A. 2007. Inconsistency in Classical Electrodynamics? Philosophy of Science 74: 253-77.

Norton, J. 1999. The Cosmological Woes of Newtonian Gravitation Theory. In H. Goenner et al. (eds.) The Expanding Worlds of General Relativity. Einstein Studies, vol.7, 271-323. . 2002. A Paradox in Newtonian Gravitation Theory II. In J. Meheus 2002, pp. 185-95.

Pais, A. 1991. Niels Bohr's Times. Oxford: Oxford University Press.

Pincock, C. 2005. Overextending Partial Structures: Idealization and Abstraction. Philosophy of Science (proceedings) 72: 1248-59.

Suppe, F. 1989 The Semantic Conception of Theories and Scientific Realism. Illinois: University of Illinois Press.

Suppes, P. 1957 Introduction to Logic. Princeton, NJ: Van Nostrand.

Thomson-Jones, M. 2006. Models and the Semantic View. Philosophy of Science 73: 524-35.

Van Fraassen, B. 1972. A Formal Approach to the Philosophy of Science. In R. Colodny (ed.) Paradigms and Paradoxes. Pittsburgh: University of Pittsburgh Press, 303-66.

Vickers, P. 2008. Frisch, Muller, and Belot on an Inconsistency in Classical Electrodynamics. British Journal for the Philosophy of Science 59(4): 767-92.

2009. Was Newtonian Cosmology Really Inconsistent? Studies in History and'Philosophy of Modern Physics 40(3): 197-208.

- (forthcoming) Reconstructing Inconsistent Scientific Theories. In P. Vickers and 0. Bueno (eds.) Is Science Inconsistent? Special issue of Synthese, forthcoming 2011.

Wilson, M. 2009. Determinism and the Mystery of the Missing Physics. British Journal for the Philosophy of Science 60: 173-93.

Peter Vickers

Centre for History and Philosophy of Science University of Leeds, UK phl4pv@leeds.ac.uk

Resumo. A abordagem semântica da representação científica está amplamente estabelecida como favorita entre os filósofos da ciência. Um dos principais objetivos dessa abordagem - a abordagem modelo-teorética (AMT) - é representar as teorias científicas como famílias de modelos, todos satisfazendo, ou 'tornando verdadeiro' um certo conjunto de condições. Entretanto, alguns autores (Brown 2002, Frisch 2005) criticaram esta abordagem alicerçando-se no fato de que algumas teorias científicas são logicamente inconsistentes, e não há modelos de um conjunto inconsistente de condições. Então deveria parecer que o AMT falha em representar teorias cientítficas inconsistentes, e isso origina questões sobre o modo geral pelo qual a abordagem reresenta teorias científicas. Em uma série de artigos (1990, 1993, 1995), e em um livro recente (2003), da Costa e French desenvolveram uma variante da AMT que denominam 'estruturas parciais'. Neste artigo, analiso esta proposta, dando atenção a duas teorias que podem ser ditas serem 'inconsistentes': a teoria do átomo de Bohr e a eletrodinâmica clássica.

Palavras-chave: Estruturas parciais, inconsistência, teorias, representação, Bohr, eletrodinâmica clássica.

Principia 13(2): 233-50 (2009). 


\section{Notes}

${ }^{1}$ Of course, the 'MTA interpreted in terms of mere description fitting' might sounds like a contradiction in terms, since it is only in 'serious-interpreting' that model-theory can be said to play a role (thanks to Martin Thomson-Jones for pointing this out). In what follows I nevertheless continue to use 'MTA' to refer to this variant of the semantic approach, merely as a label for the approach in question.

${ }^{2}$ Strictly speaking the model doesn't interpret the predicates intensionally, by providing definitions such as '_ is married to David Beckham', but merely extensionally, by providing a set of members of the domain. The intensional part of the discussion is merely used to put across the basic idea, and should not be considered an intrinsic part of the MTA.

${ }^{3}$ For further details see da Costa and French 1990; for a rigorous logical presentation see Mikenberg, da Costa and Chuaqui 1986.

${ }^{4} \mathrm{~A}$ problem arises here. I have been working on the assumption that an inconsistent theory is partially true in a structure iff each individual statement of the theory is partially true in that structure. But this means that it will be impossible to make partially true an inconsistent theory that is represented as a single conjunction of its individual claims. Perhaps the best way out of this is to say that an inconsistent theory is partially true in a structure iff each of its individual claims or conjuncts is made partially true in that structure.

${ }^{5}$ In fact, in the end it doesn't seem to be appropriate to label CED inconsistent: see §4.2, below. However, it might have been appropriate, and it is certainly useful to consider how partial structures could have accommodated CED if it was inconsistent in the way Frisch (2005) claims.

${ }^{6}$ See also Bartelborth (2000) for a Suppesian reconstruction of CED.

${ }^{7}$ Elements $f$ and $g$ are functions on Suppes' reconstruction, but they could be translated into relations. Functions and relations look the same when they are represented extensionally.

${ }^{8}$ In fact, it's not obvious how such information could be represented within the state-space approach. The best way to do it would probably be to use partial functions and even weighted functions. Thanks to Otávio Bueno for advice on this point.

${ }^{9}$ As with CED, it isn't altogether clear that it is appropriate to label Bohr's theory inconsistent. See §4.2, below.

${ }^{10}$ The later Bohr theory is another such example. See below.

${ }^{11}$ There are much better grounds for labelling the later Bohr theory 'inconsistent'. Pauli derived a contradiction in 1926 from assumptions which really were central assumptions of the theory, and had been for about eight years (roughly speaking from when Ehrenfest introduced the adiabatic principle). See Mehra and Rechenberg 1982, 507-9.

${ }^{12} \mathrm{Cf}$. Wilson 2009: '[M] uch contemporary commentary on philosophical theories of matter in the eighteenth and nineteenth centuries strikes me as greatly compromised by its inclination to assume that phrases such as 'classical mechanics' or 'the Newtonian picture' capture surgically precise meanings, when, in fact, such termirtology can be readily applied to deeply incompatible doctrines.'

${ }^{13}$ See Vickers (forthcoming) for an investigation of these issues in the context of inconsistency in science.

${ }^{14}$ Many thanks to Steven French, Otávio Bueno, Chris Pincock, Margaret Morrison and Martin Thomson-Jones for very helpful comments and discussions.

Principia 13(2): 233-50 (2009). 